\title{
Visualizing competing intersystem crossing and internal conversion with a complementary measurement
}

\author{
Yuzhu Liu, ${ }^{1, a)}$ Thomas Gerber, ${ }^{2}$ Chaochao Qin, ${ }^{3}$ Feng Jin, ${ }^{1}$ and Gregor Knopp ${ }^{2}$ \\ ${ }^{1}$ School of Physics and Optoelectronic Engineering, Nanjing University of Information Science E Technology, \\ Nanjing 210044, People's Republic of China \\ ${ }^{2}$ Paul Scherrer Institute, 5232 Villigen, Switzerland \\ ${ }^{3}$ Henan Normal University, Xinxiang 453007, People's Republic of China
}

(Received 16 November 2015; accepted 27 January 2016; published online 22 February 2016)

\begin{abstract}
A complementary measurement method based on a home-built double-sided velocity map imaging setup is introduced. This method can simultaneously obtain time-resolved photoelectron imaging and fragment ion imaging. It has been successfully applied to investigate the ultrafast dynamics of the second singlet electronically excited state $\left(\mathrm{S}_{2}\right)$ in $m$-xylene. Time-resolved photoelectron and ion signals derived from the initial populated $\mathrm{S}_{2}$ state are tracked following two-photon absorption of a pump pulse. Time-of-flight mass spectra (TOFMS) show that there are dominant parent ions and one fragment ions with methyl loss during such a process. According to the measured photoelectron images and fragment ions images, transient kinetic energy distributions and angular distributions of the generated photoelectrons and fragments are obtained and analyzed. Compared to stand-alone photoelectron imaging, the obtained fragment ion imaging is powerful for further understanding the mechanisms especially when the dissociation occurs during the pump-probe ionization. Two competing channels intersystem crossing $\mathrm{T}_{3} \leftarrow \mathrm{S}_{2}$ and internal conversion $\mathrm{S}_{1} \leftarrow \mathrm{S}_{2}$ are attributed to the deactivation of the $S_{2}$ state. A lifetime of $\sim 50$ fs for the initially excited $S_{2}$ state, of $\sim 276 \mathrm{fs}$ for the secondary populated $\mathrm{S}_{1}$ state, and of 5.76 ps for the $\mathrm{T}_{3}$ state is inferred. (C) 2016 AIP Publishing LLC. [http://dx.doi.org/10.1063/1.4942124]
\end{abstract}

\section{INTRODUCTION}

After light absorption, a molecule can undergo radiationless processes, which consist of two general types: photochemical and photophysical. ${ }^{1}$ The photophysical processes involve internal conversion due to electronic nonadiabaticity and intersystem crossing induced by spin-orbit coupling., ${ }^{2,3}$

Because the direction of the spin needs to be changed during the spin-orbit coupling process, intersystem crossing is usually a slower process than internal conversion. ${ }^{4}$ However, extensive reports show that ultrafast competing intersystem was discovered in a number of molecular systems ${ }^{5,6}$ and a wide variety of transition-metal complexes. ${ }^{7-9}$ Ultrafast intersystem crossing is an intriguing phenomenon that has posed a challenge to scientists for decades. ${ }^{10}$

Typical recent works are the following. The ultrafast intersystem crossing processes in transition-metal complexes are reported, ${ }^{7-9}$ and some of these processes are on the order of tens to hundreds of femtoseconds. The mechanism behind these processes is found to be a result of the dephasing of the photoexcited state to the phonon continuum of a different state with a significantly different transition metal-ligand distance. ${ }^{10}$ Richter and coworkers unraveled the deactivation of cytosine after UV light absorption via ab initio molecular dynamics including nonadiabatic and spin-orbit couplings on equal footing. Intersystem crossing is found to compete directly

\footnotetext{
a) Author to whom correspondence should be addressed. Electronic mail: yuzhu.liu@gmail.com
}

with internal conversion in tens of femtoseconds. ${ }^{11}$ MartinezFernandez and coworkers simulated the deactivation dynamics of photoexcited 6-thioguanine using a direct surface hopping dynamics approach. ${ }^{12}$ The results showed that 6-thioguanine is another example of a system showing ultrafast intersystem crossing that can compete with internal conversion in the same time scale. Both the spin-orbit and the dynamic couplings are important to describe realistically the excited state dynamics of 6-thioguanine.

A similar phenomenon has also been evidenced in benzene ${ }^{13,14}$ and its derivative $o$-xylene. ${ }^{15}$ Competing ultrafast intersystem crossing $T_{1} / T_{2} \leftarrow S_{1}$ was first discovered experimentally in benzene by Minns and co-workers. ${ }^{13}$ The experimental results showed that the ultrafast decay of the $S_{1}$ state in benzene was due to competing internal conversion and intersystem crossing processes and both processes occurred on a femtosecond timescale. In the previous paper, ${ }^{15}$ we reported an ultrafast competing intersystem crossing process observed in $o$-xylene via time-resolved photoelectron imaging. The interpretation challenges the widely accepted view and therefore needs justification. ${ }^{13}$

To gain more convincing evidences and features for such a challenging interpretation, we investigate another benzene derivative $m$-xylene with a complementary measurement by simultaneously obtaining photoelectron imaging and fragment ion imaging. Photoelectron imaging measures time-dependent photoelectron kinetic energy (PKE) distributions and fragment ion imaging measures time-dependent kinetic energy distributions of fragments produced in a photo-excitation process. 


\section{EXPERIMENT}

Stand-alone photoelectron imaging was and currently still is widely applied to various molecular systems. However, these studies are based on the assumption that the investigated molecules do not dissociate during the pump-probe processes or if dissociation occurs, the interference from fragments can be neglected. The simultaneous measurement of fragments together with their kinetic energy gained in the dissociation process allows associating the measured photo-electron energies with specific fragments. During photochemical reaction, the fragment ions also bring important information on photo-dynamics. Taking this into consideration, we assembled a double-sided time-resolved velocity map imaging setup, ${ }^{16}$ simultaneously collecting energy and momentum information about photoelectrons and fragment ions, a result that cannot be achieved by photoelectron imaging alone.

A sketch of the double-sided velocity map imaging setup is given in Figure 1. While it is not the exact drawing, it is shown for introduction and understanding of "double-sided imaging" (i.e., one side for ion imaging and the opposite side for photoelectron imaging). And the image is acquired with various events once different as the coincidence imaging with one event one time.

The apparatus consists of two chambers, a source chamber and a detection chamber. The two chambers are separated by a $0.5 \mathrm{~mm}$ diameter skimmer (Beam Dynamics). An additional gate valve offers an option to separate the two chambers. The source chamber has a $2050 \mathrm{l} / \mathrm{s}$ turbo molecular pump (PFEIFFER, HiPace 2300), and the detection chamber is pumped by three $67 \mathrm{l} / \mathrm{s}$ turbo molecular pumps (PFEIFFER, HiPace 80). All turbo pumps are backed together by a dry primary pump (Adixen, ACP 15). While the pressures of both the source and the detection chambers are less than $2.2 \times 10^{-7}$ Torr with the molecular beam off, they increase to $1.2 \times 10^{-5}$ Torr and $3.8 \times 10^{-7}$ Torr with the molecular beam on, respectively.

The detection system consists of two time-of-flight mass spectrometers (TOFMSs) sharing the same interaction region. Both spectrometers provide a coincident longitudinal (WileyMcLaren) and lateral velocity map image ${ }^{17}$ focus at the image plane. Each spectrometer is designed with a seven element electrodes. All the details for the design of the ion optics are shown in Fig. 2.

Velocity map imaging can be registered with a 2-stage MCP (Microchannel plate)/phosphor detector (Proxivision $\mathrm{GmbH}$ ) read out by a CCD camera (Hamamtsu Orca-03G). The high-repetition molecular beam was produced by seeding $m$-xylene (Sigma-Aldrich, 99.0\% purity) in helium buffer gas $(1.0 \mathrm{~atm})$ through a miniature valve $(\mathrm{Fa}$. Gyger) pulsed at a rate of $400 \mathrm{~Hz}$. One photoelectron/ion image typically requires approximately $120 \mathrm{~s}$ counting in CCD data acquisition.

In the present work, we apply the double-sided imaging on the study of $m$-xylene, simultaneously collecting energy and momentum information about photoelectrons and fragment ions. The TOFMS of the ions are obtained by directly recording the MCP signals of the ion side. Transient signals for all ions are measured by a home-built Labview program integrating signal intensity of the different ions of the TOFMS against the delay time simultaneously. To track ion image

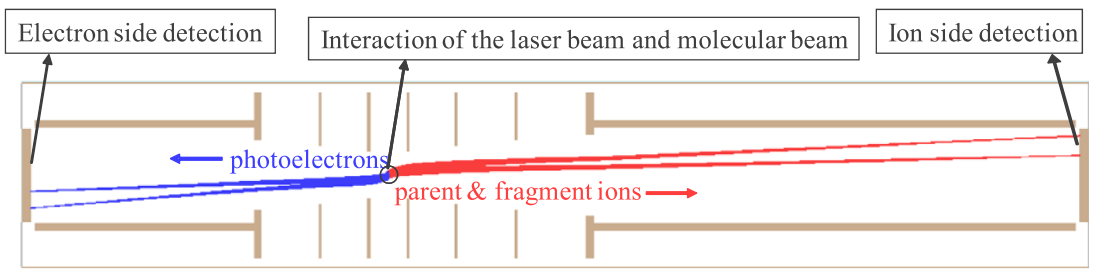

FIG. 1. A sketch of the double-sided velocity map imaging setup (please see real drawing with sizes in Fig. 2).

(a)

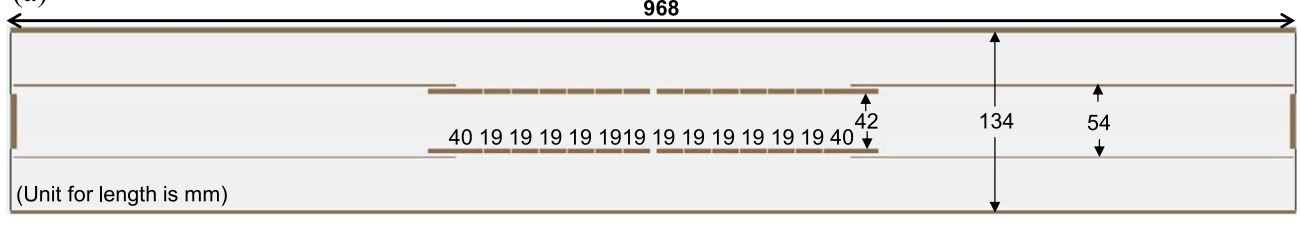

(b)

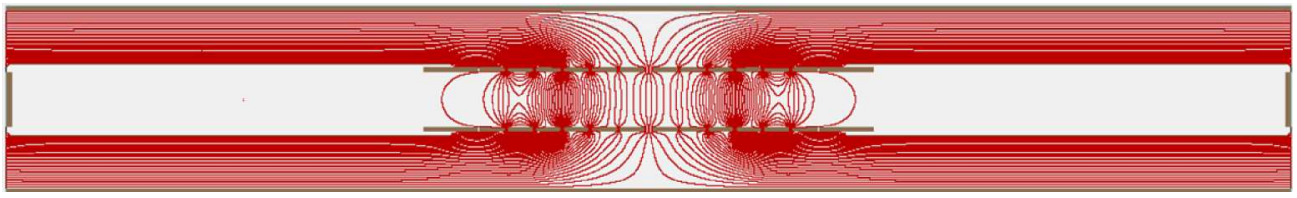

(c)

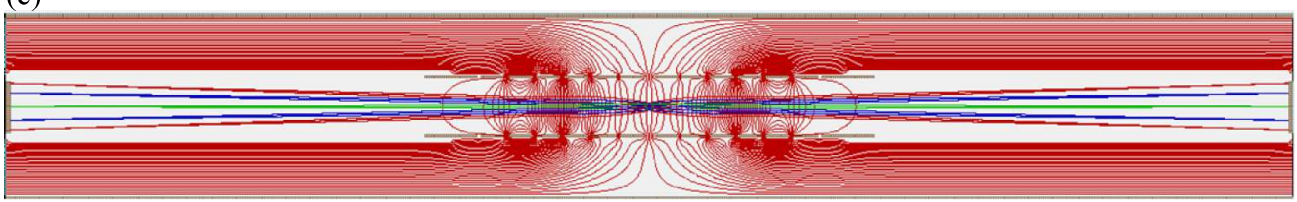

FIG. 2. (a) The cross-sectional view of the design of ion optics. The numbers are the size with unit of mm; (b) the electronic field distribution with the optimal voltages; and (c) the simulation flight trajectory of photoelectrons (left) and ions (right) with five different velocities. 
of the certain fragment ion, a high voltage pulse with $\sim 100$ ns duration forming a "mass gate" is applied to MCP to separate it from the parent ions and other possible fragment ions with different masses. The images are accumulated over 50000 shots or more. The backgrounds are removed by subtracting the images collected under the negative delay. The spectrometers are calibrated by means of an experiment on $\mathrm{CH}_{3} \mathrm{I}$ photolysis and photoionization. ${ }^{18}$

The femtosecond laser system (Clark-MXR CPA-1000) has been described in detail previously. ${ }^{19}$ The near infrared output of the laser system (at $\sim 800 \mathrm{~nm}$ ) was split into two beams with equal intensity. One part was frequency doubled to $\sim 400 \mathrm{~nm}$ in a beta barium borate crystal (BBO type I) and used as "pump" beam, while the other part passed an optical delay stage (PI, M-403.4PD) and acted as "probe." Utilizing half wave plates and thin film polarizers, both pump and probe pulses were "horizontally" polarized (with respect to the detector plane), merged with a dichroic mirror, and focused into the molecular beam chamber by a $\mathrm{f}=400 \mathrm{~mm}$ lens. Before focusing on the interaction, the laser beam diameters were reduced to $3 \mathrm{~mm}$ with a telescope. The divergence of the beam was kept as small as possible. The applied energies for pump and probe pulses were $\sim 2 \mu \mathrm{J}$ and $\sim 17 \mu \mathrm{J}$, respectively. The cross correlation width of $\sim 100$ fs was determined by recording the temporal sum frequency signal of the pump and probe pulses.

\section{RESULTS AND DISCUSSION}

\section{A. Transient parent ions and fragments}

An energy of $5.53 \mathrm{eV}$ was inferred for the lowest $\mathrm{S}_{2}$ state of $m$-xylene. ${ }^{20} \mathrm{~A}$ two-photon absorption process with $3.10 \mathrm{eV}$ photons $(400 \mathrm{~nm})$ populates the $S_{2}$ state of $m$-xylene with an excess ro-vibrational energy of $0.67 \mathrm{eV}$. Two $3.1 \mathrm{eV}$ photons cannot excite states in the $S_{3}$ state manifold having energies above $6.39 \mathrm{eV}\left(52360 \mathrm{~cm}^{-1}\right){ }^{20}$ Thus, pumping to the $S_{3}$ or higher states was not considered. The time-resolved photoelectron/ion images upon multi-photon ionization starting from the $S_{2}$ and possibly secondary populated states are acquired at different delays between pump and probe. The TOFMS with the pump beam and with the pump-probe ionization are given in Figs. 3(a) and 3(b), respectively.

The ionization potential of $m$-xylene is determined to 8.57 $\mathrm{eV}$ as reported. ${ }^{22}$ An applied pump with 3-photon $(\sim 9.30 \mathrm{eV})$ or higher excitation at $400 \mathrm{~nm}$ is likely to ionize $m$-xylene molecules. To avoid such a possibility, the pump beam with low energy is chosen in the current experiment. According to Fig. 3(a), with the interaction of the pump beam only, the generated ion signal is extremely weak, indicating that the possibility of 3-photon or higher excitation can be neglected in the measurement. And with the probe only, the signal is similarly weak. Nevertheless, combining the pump together with the probe beam at the specific delay time (i.e., within the lifetime), we obtained enhanced signals as shown in Fig. 3(b).

TOFMS shown in Fig. 3(b) indicate that there are one dominant parent-ion $\mathrm{C}_{6} \mathrm{H}_{4}\left(\mathrm{CH}_{3}\right)_{2}^{+}$and one fragment ion $\mathrm{C}_{6} \mathrm{H}_{4} \mathrm{CH}_{3}{ }^{+}$with $\mathrm{CH}_{3}$ loss by fragmentation. The signal intensity of the fragment ion is low, and the ratio of the
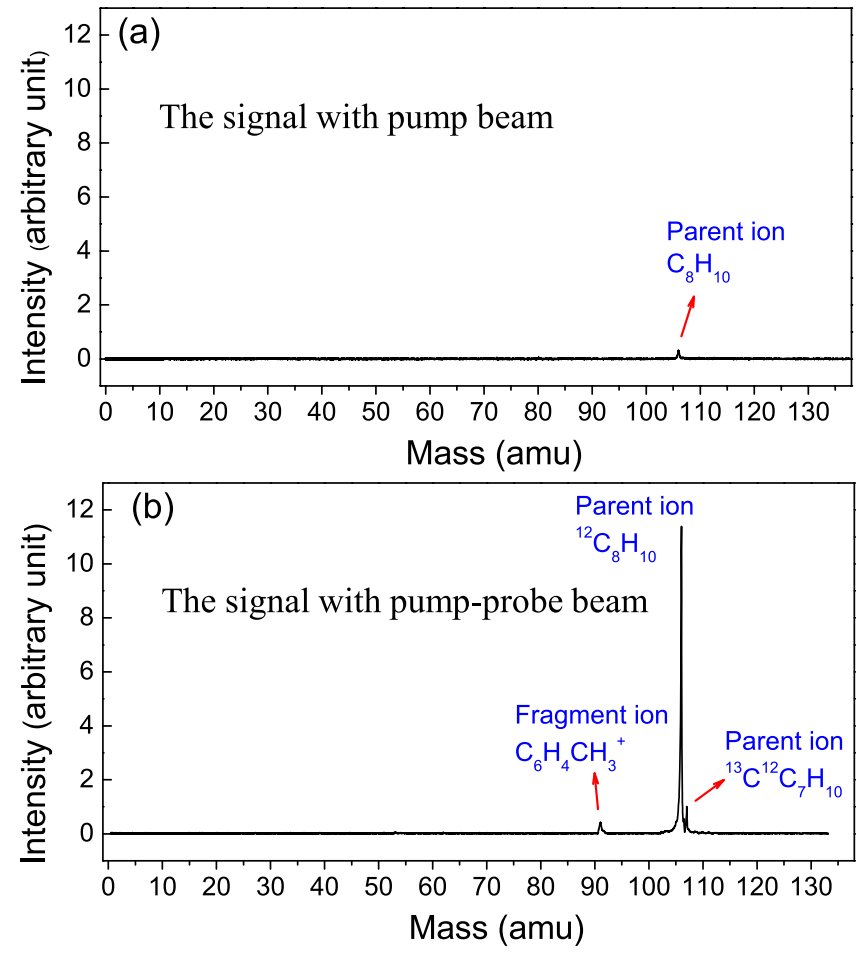

FIG. 3. The TOFMS signal of photoionization/fragmentation with the pump beam (a) and with pump and probe beams at the delay time of zero (b).

fragment ion to the parent ion is roughly $5 \%$. The power of the probe beam in the measurements is too weak to probe the neutral fragment. The appearance of fragment ion $\mathrm{C}_{6} \mathrm{H}_{4} \mathrm{CH}_{3}{ }^{+}$ is due to the dissociation of the parent ions after pump-probe ionization during the flight process to the detector.

Figs. 4(a) and 4(b) show transient signals of the fragment-ion $\mathrm{C}_{6} \mathrm{H}_{4} \mathrm{CH}_{3}{ }^{+}$due to $\mathrm{CH}_{3}$ loss and the parent ions $\mathrm{C}_{6} \mathrm{H}_{4}\left(\mathrm{CH}_{3}\right)_{2}^{+}$, respectively. The temporal behaviour for both parent and fragment ions was fitted to exponential functions convoluted with a 100 fs wide Gaussian instrument response function. For the fragment ions $\mathrm{C}_{6} \mathrm{H}_{4} \mathrm{CH}_{3}{ }^{+}$, three components with time constants of $\tau_{1}=\sim 50 \mathrm{fs}, \tau_{2}=210$ $( \pm 32)$ fs, and $\tau_{3}=5.5( \pm 0.8)$ ps were observed. However, only two corresponding components appeared on parent ion. The fitted time constants for these two component were $\tau_{1}=52( \pm 5)$ fs and $\tau_{3}=4.6( \pm 0.6) \mathrm{ps}$, respectively. The differences on time constants $\tau_{1}$ and $\tau_{3}$ between the parent-ion and fragment-ion were in the order of the fitting error.

The fragment ions are from the dissociation of the parent ions. Thus they are expected to have the similar trend. Interestingly, the second component with time constant of $\tau_{2}$ is missing for parent ions in contrast to that for the fragment ions. For clarity, the difference of the signal of the fragment ions and the parent ions after normalization is given as shown in Fig. 4(d). It is clear that the component with $\tau_{2}$ fits quite well with the difference of the signal of the fragment ions and the parent ions and why is the component with $\tau_{2}$ missing for parents but resistant in fragments. It can be proposed that the parent ions from this component have dissociated during the flight before they are captured by the MCP detector. Such a proposition is supported by the observed transient photoelectron signal as shown in Fig. 4(c). The fitting results 


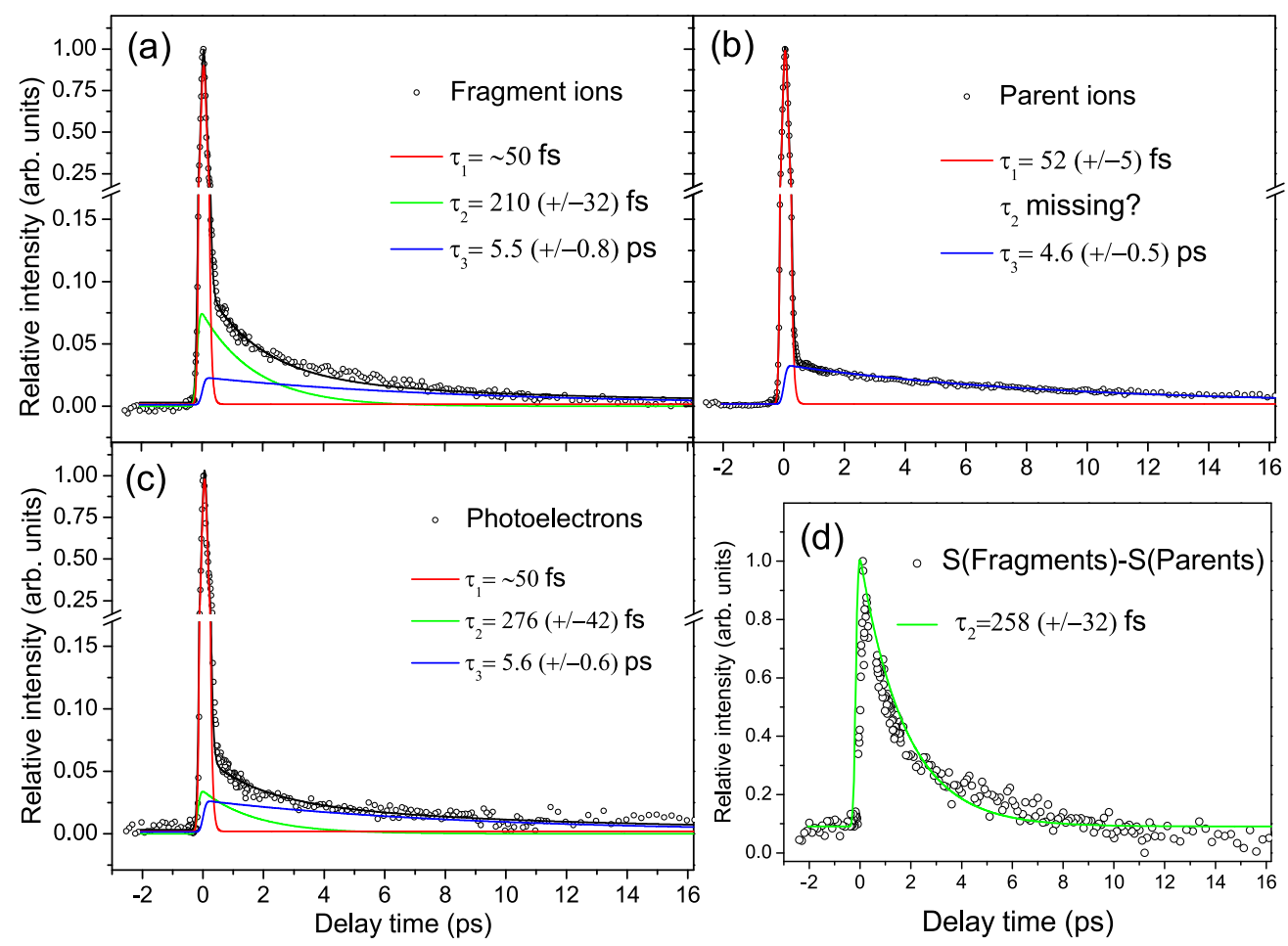

FIG. 4. Transient signal of (a) the fragment-ion $\mathrm{C}_{6} \mathrm{H}_{4} \mathrm{CH}_{3}{ }^{+}$, (b) the parent ion $\mathrm{C}_{6} \mathrm{H}_{4}\left(\mathrm{CH}_{3}\right)_{2}{ }^{+}$, (c) the total photoelectron signal, and (d) the difference of the signal of the fragment ions and the parent ions after normalization.

for electrons are very similar to that for the fragment ions. Three fitted constants $\tau_{1}=\sim 50 \mathrm{fs}, \tau_{2}=276( \pm 42) \mathrm{fs}$, and $\tau_{3}=5.6( \pm 0.6) \mathrm{ps}$ are obtained, respectively. The fitted results are much similar to that for the fragment ions with small difference within fitting error. Without secondary dissociation after ionization, the transient signal of the electrons and the parent ions are expected to be exactly the same. The only reasonable explanation for the findings is that the parent ions from this component undergo fragmentation with methyl loss before the ions leave the acceleration field.

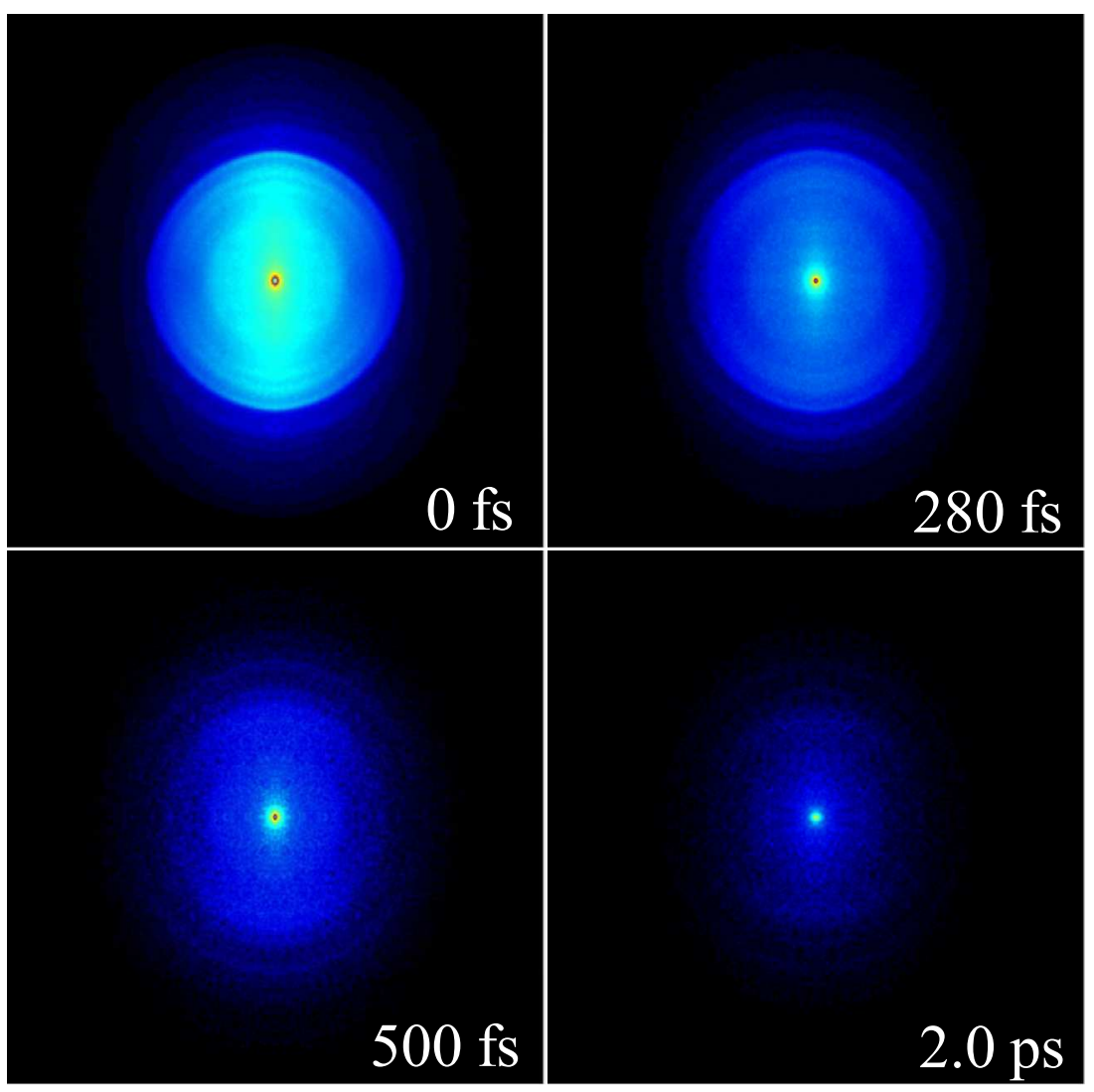

FIG. 5. Time-resolved photoelectron imaging at the delay times of $0 \mathrm{fs}$, $280 \mathrm{fs}, 500 \mathrm{fs}$, and $2.0 \mathrm{ps}$. 


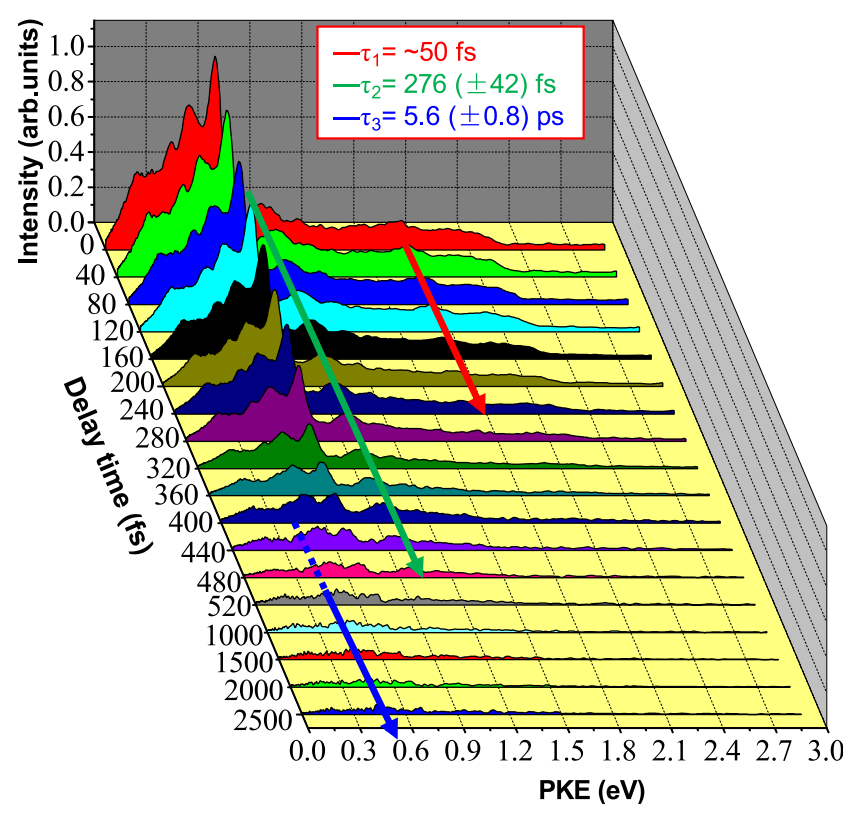

FIG. 6. Time-resolved PKE distributions extracted from the time-resolved photoelectron images. The three arrows indicate the three different time scales.

From the complementary measurements, the detailed discussions given below based on photoelectrons as well as fragments show that the component with time constant of $\tau_{2}$ is from the ionization of the secondary populated $S_{1}$ state with the high vibrational excitation energy. With the assumption that the vibrational energy remains conserved in ionization, the parent ions from such a component are also expected to have high vibrational excitation. Thus the parent ions with high vibrational energy from such a channel are more likely to undergo vibrational dissociation.

\section{B. Time-resolved photoelectron imaging}

Fig. 5 shows images in which photoelectrons with different kinetic energy are displayed at various typical delay times of $0 \mathrm{fs}, 280 \mathrm{fs}, 500 \mathrm{fs}$, and $2.0 \mathrm{ps}$. The corresponding PKE distributions can be inferred from the measured photoelectron images by lin-Base ${ }^{21}$ as a function of energy and of the pump-probe time delay (Fig. 6). The time-resolved PKE distributions provide transients with three distinct relaxation time scales indicated by the three arrows shown in Fig. 6.

The total photoelectron intensity as a function of the pump-probe time delay is shown in Fig. 4(c). The temporal behaviour can be well fitted to three exponential decays, convoluted with a $100 \mathrm{fs}$ wide Gaussian instrument response function. Three distinct lifetimes of $\tau_{1}=\sim 50 \mathrm{fs}, \tau_{2}=276$ $( \pm 42)$ fs, and $\tau_{3}=5.6( \pm 0.6)$ ps are determined from the least square fit. The three constants correspond to the time scale of the three PKE-bands indicated with arrows in Fig. 6. The observed PKEs depend on the energy difference between the excited intermediate states and the final ion state. In this respect, time dependent photoelectron imaging allows for simultaneous monitoring of the electronic and ro-vibrational excited-state dynamics. ${ }^{23,24}$

Three typical PKE distributions at $\mathrm{t}=0 \mathrm{fs}, 280 \mathrm{fs}$, and 2000 fs are shown together in Fig. 7(a) for closer inspection. For reference, an energy excitation scheme including the electronically ground, excited, and ion states of $m$-xylene is sketched in Fig. 7(b). In Fig. 7(a), the green arrow at $0.73 \mathrm{eV}$ and the blue arrow at $2.28 \mathrm{eV}$ indicate the maximum possible electron energy, i.e., the excess energy above the adiabatic ionization limit, after two- and three-photon ionization using an $800 \mathrm{~nm}$ probe beam, the ionization energy of $m$-xylene being $8.57 \mathrm{eV}$. It is noted that $m$-xylene molecules have at most $\mathrm{C}_{2 v}$ symmetry considering methyl groups as point masses. ${ }^{25,26}$ Under this assumption, the vibronic states of $m$-xylene have $\mathrm{A}_{1}, \mathrm{~A}_{2}, \mathrm{~B}_{1}$, or $\mathrm{B}_{2}$ symmetry in the neutral and ionic states. No priority for particular excitation and ionization bands can be derived from basic group theoretical considerations alone.

As shown in Fig. 7(a), nine peaks centred at $0.18 \mathrm{eV}, 0.47$ $\mathrm{eV}, 0.64 \mathrm{eV}, 0.91 \mathrm{eV}, 1.10 \mathrm{eV}, 1.46 \mathrm{eV}, 1.71 \mathrm{eV}, 1.97 \mathrm{eV}$, and $2.17 \mathrm{eV}$ are resolved in the PKE distribution at $\mathrm{t}=0 \mathrm{fs}$. We assign them as peak \#1 to peak \#9 in the order of increasing kinetic energy. All peak intensities gradually decrease with time. At a delay of $280 \mathrm{fs}$, only peak \#1, \#4, and \#5 remain.

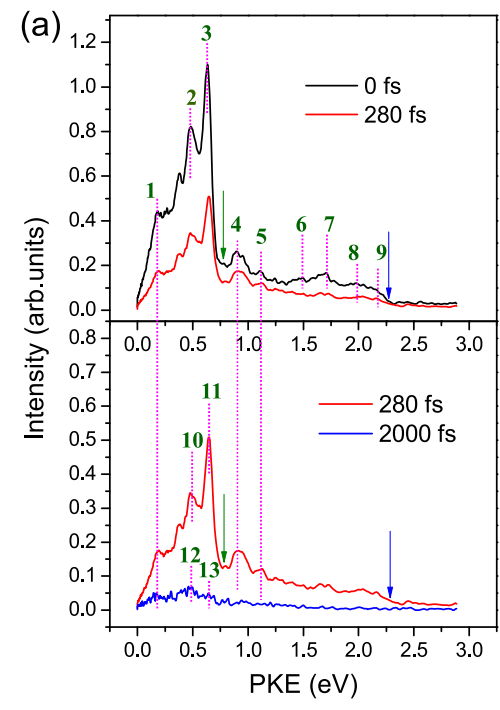

(b)

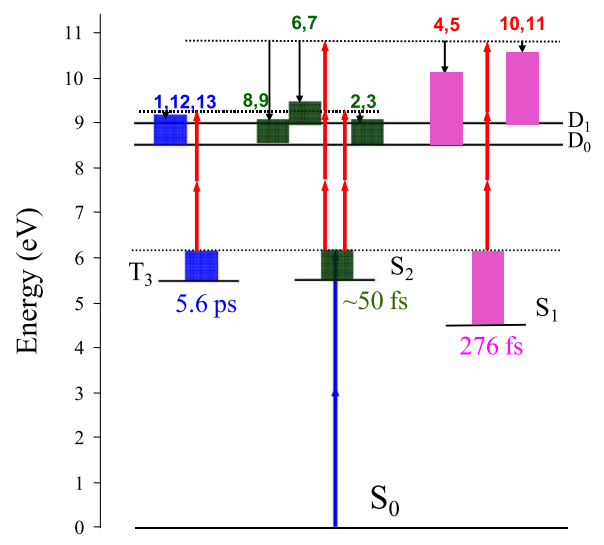

FIG. 7. (a) Three typical PKE distributions at $\mathrm{t}=0 \mathrm{fs}, 280 \mathrm{fs}$, and $2000 \mathrm{fs}$. The green arrow at $0.73 \mathrm{eV}$ and blue arrow at $2.28 \mathrm{eV}$ indicate the maximum electron energy by two-photon and threephoton absorption of probe beam at 800 $\mathrm{nm}$ after two-photon excitation of the $\mathrm{S}_{2}$ state at $400 \mathrm{~nm}$. (b) Energy excitation scheme of the ground, excited, and ionic states of $m$-xylene. 
The other peaks reduce to the noise level at $\sim 280 \mathrm{fs}$. At this time, some new peaks (\#10 and \#11) appear around the positions of peak \#2 and \#3. Peak number 2, 3, 6, 7, 8, and 9 can be attributed to probe pulse ionization of the initial populated $S_{2}$ state (Fig. 7(b)).

Six peaks share a common intensity decay that corresponds to the fitted time constant of $\tau_{1}=\sim 50 \mathrm{fs}$, as inferred from the integral photoelectron signal. The ultrafast intensity drop denotes a quick depopulation of the initially excited $S_{2}$ state. According to the scheme in Fig. 7(b), the most intense peaks at $\mathrm{t}=0 \mathrm{fs}$ are $\# 2$ at $0.47 \mathrm{eV}$ and \#3 at $0.64 \mathrm{eV}$, and they are due to the ionization of the $\mathrm{S}_{2}$ state to $\mathrm{D}_{0}$ by a two-photon absorption of the probe beam. The energy difference between the two peaks is calculated $(\Delta=0.64-0.47=0.17 \mathrm{eV})$. And it corresponds well to the vibrational splitting in ionic state. Peaks \#6, \#7, \#8, and \#9 are beyond the limit of the two-photo ionization process. The energy difference is $1.50 \mathrm{eV}$ between peak \#2 and \#8 and 1.53 $\mathrm{eV}$ between \#3 and \#9. Both differences are close to the probe photon energy $(1.55 \mathrm{eV})$. Moreover, peaks 8 and 9 have the same temporal evolution as peaks 2 and 3 . Thus we conclude that peaks 8 and 9 stem from ionization of the $S_{2}$ state to $D_{0}$ by three-photon absorption. The energy difference of $0.20 \mathrm{eV}$ between peaks 8 and 9 can again be interpreted as the cationic vibrational splitting. By three-photon absorption, both the $\mathrm{D}_{0}$ and $\mathrm{D}_{1}$ ionic states become accessible. The difference of 0.51 $\mathrm{eV}$ between the peaks of \#6 and \#8, and $0.46 \mathrm{eV}$ between $\# 7$ and \#9, matches the energy difference between the $\mathrm{D}_{0}$ and $\mathrm{D}_{1}$ state of $0.50 \mathrm{eV} .{ }^{27}$ Thus it is reasonable to attribute peaks \#6 and \#7 to the ionization of the $S_{2}$ state to ion $D_{1}$ state by three-photon absorption of the probe beam. Due to the existence of the cationic vibrational splitting, all peaks appear in pairs, e.g., \#2 and \#3, \#6 and \#7, and \#8 and \#9.

As shown in Fig. 7(a), the peaks \#1, \#4, and \#5 persist for longer time delays. Two new peaks, centered at $0.47 \mathrm{eV}$ and $0.64 \mathrm{eV}$, are assigned as \#10 and \#11, respectively, and they become visible after $\sim 280$ fs delay time. At zero delay, the latter peaks are overlaid by peaks \#2 and \#3 and therefore not visible. At a delay of $280 \mathrm{fs}$, five peaks (\#1, \#10, \#11, \#4, and \#5) can be observed in the PKE distributions which predominantly correspond to the ionization of secondary populated states.

Further study shows that peak \#4 and peak \#5 exhibit a similar time dependency as peaks 10 and 11 . The exponential decay rate constant of all four peaks was fitted to be very close to $\tau_{2}=\sim 276 \mathrm{fs}$. According to the energy term scheme in Fig. 7(b), these four peaks are due to the ionization from the $S_{1}$ state after internal conversion from $S_{2}$. The origin of the $S_{1}$ state has been measured to be at $4.58 \mathrm{eV}$ by REMPI. ${ }^{28}$ After internal conversion from the $S_{2}$ to the $S_{1}$ state, the overall ro-vibrational excitation energy in $S_{1}$ is $\sim 1.62 \mathrm{eV}$. As shown in Fig. 7(b), with the assumption that the ro-vibrational energy remains conserved in ionization, a two-photon absorption of the probe beam is most unlikely.

The difference of $0.44 \mathrm{eV}$ between peak \#4 and \#10, and $0.46 \mathrm{eV}$ between 5 and 11, matches the energy difference of $0.50 \mathrm{eV}$ between the $\mathrm{D}_{0}$ and $\mathrm{D}_{1}$ states. This is similar to the \#6, \#8 and \#7, \#9 peak combinations regarding $S_{2}$. Considering the energy level of $S_{1}$ state, the peaks \#4, \#5 and
$\# 11$, \#12 relate to the $S_{1} \rightarrow D_{0}$ and $S_{1} \rightarrow D_{1}$ state transition, respectively. The difference of 0.17 and $0.19 \mathrm{eV}$ between peaks 4 and 5 and between peaks 10 and 11 is due to the vibrational splitting in the cation, mentioned before.

As shown in Fig. 5, all peaks related to the $S_{1}$ state ionization vanish within a few hundred fs. By exponential data fitting, the lifetime of the hot vibrational $S_{1}$ state has been determined to be $\sim 276 \mathrm{fs}$. It is consistent with the fast decay time of 550 fs observed for high vibronic states (with excess energy $>4000 \mathrm{~cm}^{-1}$ ) in the $S_{1}$ state of benzene, which can be explained by a conical intersection of the $S_{1}$ and $S_{0}$ potential-energy surfaces. ${ }^{29}$

As evident in Fig. 7(a) at delay time of $2.0 \mathrm{ps}$, a third longer-lived component was observed with a decay constant of $\sim 5.6$ ps. Mainly the three peaks, number 1, 12, and 13, contribute to this component (Fig. 7(a)). Energetic considerations (see Fig. 7(b)) suggest that these peaks can be attributed to the ionization from a triplet state. The first three triplet states are measured to be $3.7-3.8 \mathrm{eV}$ for $\mathrm{T}_{1}, 4.6 \mathrm{eV}$ for $\mathrm{T}_{2}$, and $5.5 \mathrm{eV}$ for $\mathrm{T}_{3}$ by Yamamoto and co-workers, respectively. ${ }^{30}$ Competing ultrafast intersystem crossing $\mathrm{T}_{1} / \mathrm{T}_{2} \leftarrow \mathrm{S}_{1}$ was first discovered experimentally in benzene by Minns and co-workers. ${ }^{13}$ They explained that the observed ultrafast intersystem crossing is due to the near degeneracy of the involved singlet and triplet states over a wide range of geometries traversed by the wave packet as it moves away from the Franck-Condon region. For $m$-xylene, the position of the $\mathrm{T}_{3}$ state at $5.5 \mathrm{eV}$ is also very close to the $\mathrm{S}_{2}$ state at $5.53 \mathrm{eV}$.

The PKE distribution from ionization of the $T_{3}$ state is thus expected to have no shift compared with that for the $S_{2}$ state under the same 2-photon ionization mechanism. The peaks 1, 10, and 13 at $2.0 \mathrm{ps}$ are centred at $0.18 \mathrm{eV}$, $0.46 \mathrm{eV}$, and $0.64 \mathrm{eV}$, respectively. The peaks with the same ionization mechanism (ionization to $\mathrm{D}_{0}$ by two-photon-probe ionization) from the $S_{2}$ state are peaks \#2 and \#3, at 0.47 $\mathrm{eV}$ and $0.64 \mathrm{eV}$, respectively. The difference between peaks $\# 12$ and \#2 and between peaks \#13 and \#3 is zero or very close to zero, fostering the attribution of peak \#12 and of peak \#13 to ionization from the $T_{3}$ state as depicted in Fig. 7(b). The peak \#1 exhibits the same time evolution trend as the peaks \#12 and \#13, which suggests that it originates from the same intermediate triplet state. Three-photon ionization peaks from the secondary populated $T_{3}$ state have not been clearly observed. Some faint features beyond the green arrow in Fig. 7(a) may be attributed to this three photo ionization process but a definite assignment is not possible due to the low intensities.

\section{Time-resolved fragment-ion imaging}

As given in Section III A, the measurements on transient signal of fragments and parent ions show that the fragment ions have the same time constant as the photoelectrons even though one component with time constant of $\tau_{2}$ is missing for the parent ions. As we proposed above, the reason is that the parent ions from this component have dissociated during the flight before they reached the MCP. In Section III B, we have given attributions to these three components based on the energy of 


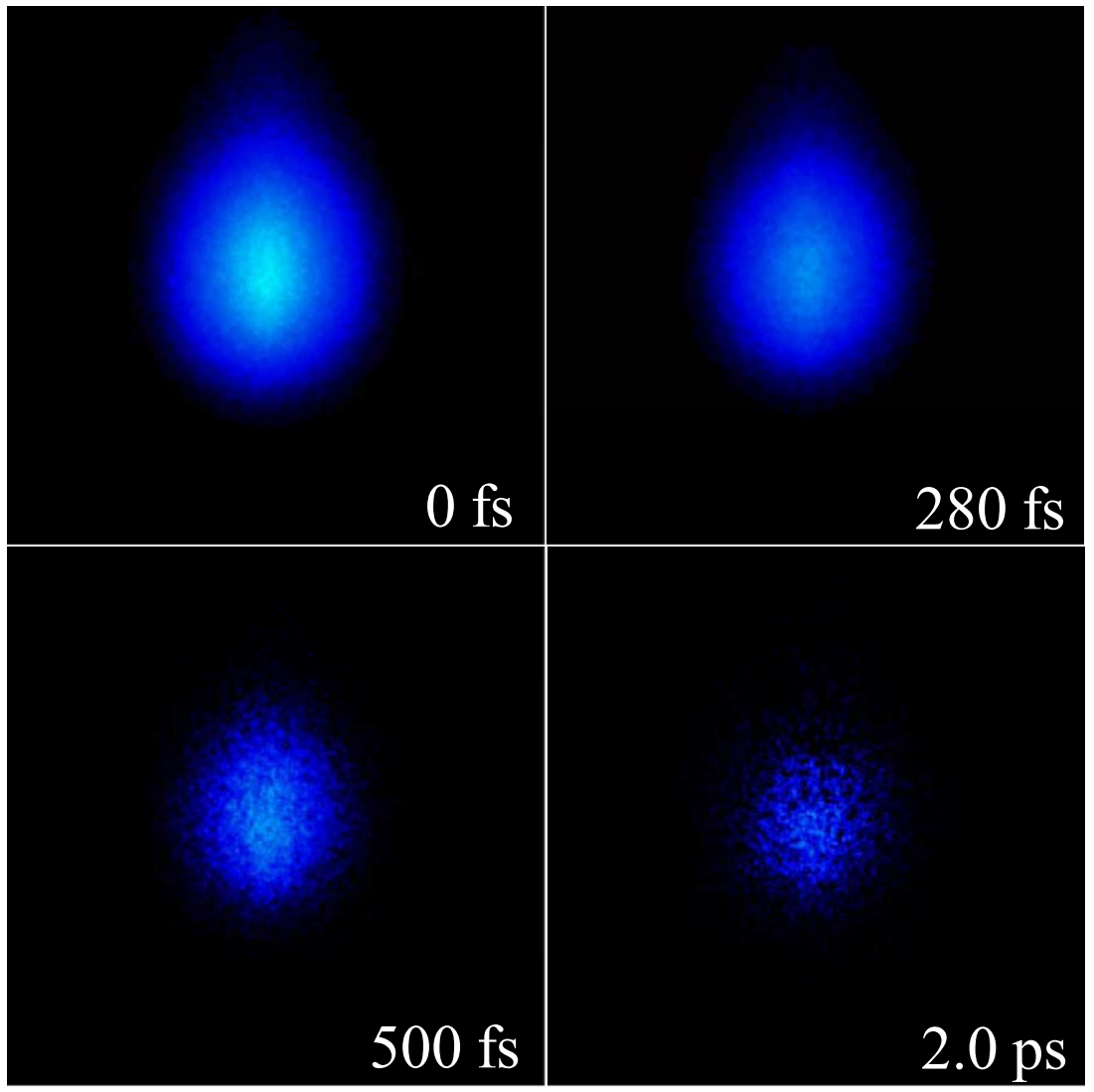

FIG. 8. Transient ion images of the fragment-ion $\mathrm{C}_{6} \mathrm{H}_{4} \mathrm{CH}_{3}{ }^{+}$at the delay time of $0 \mathrm{fs}, 280 \mathrm{fs}, 500 \mathrm{fs}$, and $2.0 \mathrm{ps}$. the ejected photoelectrons. The component with time constant of $\tau_{1}=\sim 50$ fs is from the ionization of initial populated $S_{2}$ state. And the second one with $\tau_{2}=\sim 276 \mathrm{fs}$ and the third one with $\tau_{3}=\sim 5.6 \mathrm{ps}$ are due to the ionization from the secondary populated $S_{1}$ state and $T_{3}$ state, respectively. Due to the energy conversion, the secondary populated $S_{1}$ state is highly vibrational excited with the vibrational excitation energy of $\sim 1.62 \mathrm{eV}$. With the assumption that the vibrational energy remains conserved in ionization, the parent ions from this second component are also expected to have high vibrational excitation energy in the order of $\sim 1.62 \mathrm{eV}$. For the first component from the $\mathrm{S}_{2}$ state and the third component from the $\mathrm{T}_{3}$ state, the vibrational energies are much lower. The current transient ion measurement indicates that the second component ascribed from the vibrational hot $S_{1}$ state is missing in the parent ions displayed in Fig. 4(b). And it is "missing" because of the dissociation of the parent ions prior to detection. The dissociation rate is fast compared with the flight time of $11.9 \mu$ s recorded by TOFMS, thus such a channel cannot be observed for the detector.

Further examination of the fragment ions shows transient fragment ion images of $\mathrm{C}_{6} \mathrm{H}_{4} \mathrm{CH}_{3}{ }^{+}$are simultaneously recorded by the opposite side as a function of the delay time, as shown in Fig. 8. From the ion images, the kinetic energy distributions for the fragments ions can be inferred. ${ }^{16}$ For ease of comparison, all the kinetic energy distributions at the different delay time are normalized to the strongest signal as shown in Fig. 9.

As we discussed in Section III B, there are three populated states with different lifetimes and vibrational energies. The vibrational energies naturally produce the fragment ions with different translational kinetic energy, so at different probe delays, it can be expected to find different translational kinetic energy distributions in the superimposed signal as the different populations decay at different rates. Time-resolved translational kinetic energy of fragment ion $\mathrm{C}_{6} \mathrm{H}_{4} \mathrm{CH}_{3}{ }^{+}$shown in Fig. 9 characterizes such transient changes in two aspects. First, the translational kinetic energy distribution of fragment ions $\mathrm{C}_{6} \mathrm{H}_{4} \mathrm{CH}_{3}{ }^{+}$becomes narrow against the delay time. Here the full width at half maximum decreases from $0.102 \mathrm{eV}$ at $0 \mathrm{fs}, 0.086 \mathrm{eV}$ at $280 \mathrm{fs}$, and $0.067 \mathrm{eV}$ at $500 \mathrm{fs}$ to $0.043 \mathrm{eV}$ at $2.0 \mathrm{ps}$, gradually. The discussion based on photoelectron signal has indicated that $\mathrm{S}_{1} \leftarrow \mathrm{S}_{2}$ internal conversion and

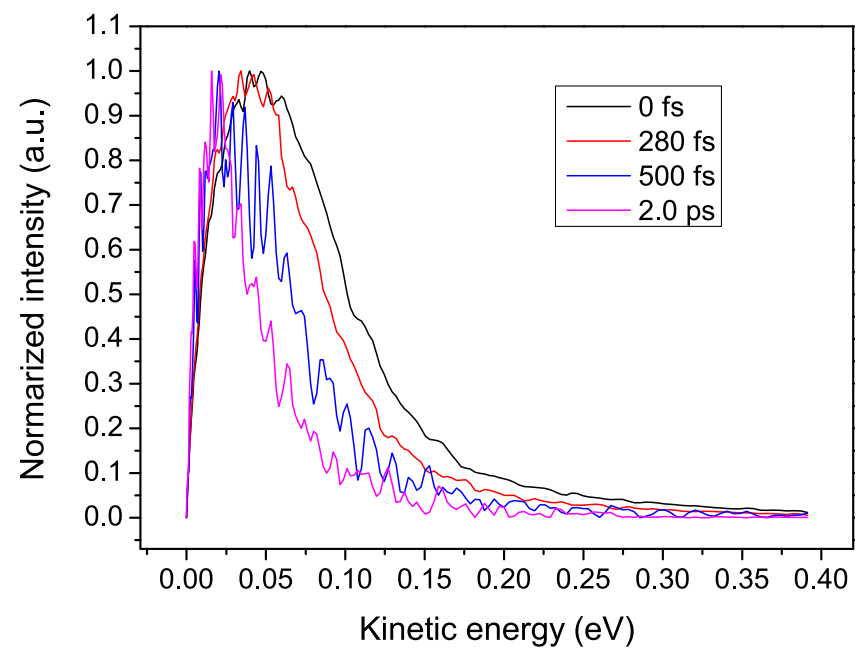

FIG. 9. Time-resolved kinetic energy distributions for the fragment-ion $\mathrm{C}_{6} \mathrm{H}_{4} \mathrm{CH}_{3}{ }^{+}$at the different delay time. 
$\mathrm{T}_{3} \leftarrow \mathrm{S}_{2}$ intersystem crossing occur on tens of femtoseconds. At the very beginning, three states (the initial $S_{2}$ state, the secondary populated $S_{1}$ state by internal conversion, and the secondary populated $\mathrm{T}_{3}$ state by intersystem crossing) are populated. As the time delay, the initial $S_{2}$ state depopulated completely with lifetime of $\tau_{1}=\sim 50$ fs. The secondary populated $\mathrm{S}_{1}$ and $\mathrm{T}_{3}$ states also decay with lifetime of $\sim 276$ fs and $\sim 5.6 \mathrm{ps}$, respectively. Thus, as the time delay changes from $0 \mathrm{fs}$ to $2 \mathrm{ps}$, the populated states deactivate gradually with different lifetime, which can explain the narrow kinetic energy distribution of the superimposed signal. Second, the peak position of the translational kinetic energy distribution decreases with the delay time. After absorption of the pump photons, two competing relaxation channels of the $S_{2}$ state were observed and assigned to the $S_{1} \leftarrow S_{2}$ internal conversion and $T_{3} \leftarrow S_{2}$ intersystem crossing, respectively. Following radiationless electronic relaxation from the upper electronic $\mathrm{S}_{2}$ state to the lower states, more energy is distributed to the lower electronically excited states. Under the assumption that vibrational level should be conserved after ionization by the probe beam, the cations prior to the dissociation are expected to have higher vibrational energy from the ionization of the lower secondary populated states. Therefore, the observed red-shift of the translational kinetic energy distribution can be explained by the fact that more available energy is distributed to the vibrational states accompanying radiationless electronic relaxation with the delay time. The complementary measurements of the fragment ions support the attribution from the view of the photoelectrons given in Section III B.

Benzene exhibits a sharp, apparently anomalous, fluorescence decrease approximately $3000 \mathrm{~cm}^{-1}$ above the origin of the $S_{1}$ state $\left({ }^{1} B_{2 u}\right)$ for which no satisfying explanation has appeared. This nonradiative channel has been known as "channel three." It was first observed by Callomon et al. from the diffuseness of the vibronic bands. ${ }^{31}$ Consistent with the highly reduced excited state lifetime, they observed only diffuse vibronic bands in the measured spectra. Since they thought that none of the standard processes (IC, ISC or IVR) were responsible, this unknown phenomenon was named "channel three" and remained mysterious for a long time. ${ }^{32-35}$ In previous work, we investigated the low vibronic $S_{1}$ state of $o$-xylene with one-photon pump at $266 \mathrm{~nm}$. The lifetime of the low vibronic $S_{1}$ state with vibronic energy of $0.02 \mathrm{eV}$ is much longer, extrapolated to $\sim 12.7 \mathrm{~ns}^{36}$ The great differences of lifetime of different vibronic $S_{1}$ state are discovered and they are proposed to be attributed to their different radiationless dynamics. Later, by measuring photoelectron spectra to several picoseconds of pump-probe delay and an energy resolution better than 0.01 at $1 \mathrm{eV}$, provided by our new experimental setup, we found that an additional electronic state is involved in the deactivation of the $S_{2}$ state. This new competing relaxation channel has been assigned to $\mathrm{T}_{3} \leftarrow \mathrm{S}_{2}$ ISC according to the experimental data and theoretical considerations. ${ }^{15}$ And the lifetime of the high vibronic $S_{1}$ state with vibronic energy of $1.57 \mathrm{eV}$ is much shorter, fitted to $\sim 540 \mathrm{fs}$. In the current work, we measure photoelectron imaging with simultaneous obtaining fragment ion imaging on the $m$-xylene, an isomer of $o$-xylene. More convincing evidence for the deactivation of the
$\mathrm{S}_{2}$ state is obtained from such a complementary measurement. And the lifetime of the high vibronic $S_{1}$ state with vibronic energy of $1.62 \mathrm{eV}$ is measured to $\sim 276 \mathrm{fs}$. Beyond the complementary method and ultrafast dynamics of the $S_{2}$ state, it is hoped that the results via all the above measurements are also helpful for further understanding of "channel three" region on benzene and its derivatives.

\section{CONCLUSIONS}

We assembled a double-sided time-resolved VMI setup, simultaneously collecting energy and momentum information about electrons and coincident fragment ions. In this paper, the double-sided velocity map imaging setup was successfully applied to investigate the competing ultrafast intersystem crossing and internal conversion dynamics of the $S_{2}$ in $m$-xylene. Time-resolved photoelectron imaging and fragment ion imaging following the initially populated $S_{2}$ state upon two-photon absorption of $400 \mathrm{~nm}$ are obtained simultaneously. Transient kinetic energy distributions for the generated photoelectrons and fragment ions are analyzed and discussed. Competing intersystem crossing and internal conversion were visualized from the measured photoelectrons as well as fragment ions.

The lifetime of the $S_{2}$ state is determined to be $\sim 50$ fs according to the decay of the measured photoelectron intensities. A fast internal conversion from the $S_{2}$ to the hot vibrational $S_{1}$ state with vibrational excitation energy of $\sim 1.62 \mathrm{eV}$ is evidenced. It is found that the parent ions from the ionization of the hot vibrational $S_{1}$ state are "missing" from the detection. It is because the parent ions from this component have already dissociated during the flight process due to the high vibrational energy. Such findings may bring a discussion on whether the investigations on molecular ultrafast dynamics via time-resolved measurements based on parent ions alone are accurate or not.

Moreover, a competing intersystem crossing process has also been observed in the experiments, which has been assigned to the $T_{3} \leftarrow S_{2}$. This process occurs on a similar femtosecond timescale as the process $\mathrm{S}_{1} \leftarrow \mathrm{S}_{2}$. For $m$-xylene, the energy level for $S_{2}$ and $T_{3}$ is nearly isoenergetic. As we discussed in the previous paper on $o$-xylene, such an ultrafast intersystem crossing can be explained by that the spin-orbit coupling is highly effective due to the near degeneracy of the involved singlet and triplet states. The lifetime of the secondary populated $\mathrm{T}_{3}$ state has been determined to be $\sim 5.6 \mathrm{ps}$.

\section{ACKNOWLEDGMENTS}

This work was supported by the National Natural Science Foundation of China (Grant Nos. 11304157 and U1404112), the Swiss National Centre of Competence in Research, Molecular Ultrafast Science and Technology (NCCR-MUST), Six talent peaks project in Jiangsu Province (Grant No. JNHB011), and the Swiss Federal Office of Energy (BFE).

\footnotetext{
${ }^{1}$ A. H. Zewail, Science 307, 558 (2005).

${ }^{2}$ I. V. Hertel and W. Radloff, Rep. Prog. Phys. 69, 1897 (2006)

${ }^{3}$ A. H. Zewail, Angew. Chem., Int. Ed. 39, 2586 (2000).
} 
${ }^{4}$ D. A. McQuarrie and J. D. Simon, Physical Chemistry: A Molecular Approach (University Science Books, 1997).

${ }^{5}$ J. S. Zugazagoitia, E. Collado-Fregoso, E. F. Plaza-Medina, and J. Peon, J. Phys. Chem. A 113, 805 (2009).

${ }^{6}$ J. S. Zugazagoitia, C. X. Almora-Diaz, and J. Peon, J. Phys. Chem. A 112, 358 (2008).

${ }^{7}$ Y. Ogawa, S. Koshihara, K. Koshino, T. Ogawa, C. Urano, and H. Takagi, Phys. Rev. Lett. 84, 3181 (2000).

${ }^{8}$ W. Gawelda, V. T. Pham, M. Benfatto, Y. Zaushitsyn, M. Kaiser, D. Grolimund, S. L. Johnson, R. Abela, A. Hauser, Ch. Bressler, and M. Chergui, Phys. Rev. Lett. 98, 057401 (2007).

${ }^{9}$ Ch. Bressler, C. Milne, V.-T. Pham, A. ElNahhas, R. M. van der Veen, W. Gawelda, S. Johnson, P. Beaud, D. Grolimund, M. Kaiser, C. N. Borca, G. Ingold, R. Abela, and M. Chergui, Science 323, 489 (2009).

${ }^{10}$ M. van Veenendaal, J. Chang, and A. J. Fedro, Phys. Rev. Lett. 104, 067401 (2010).

${ }^{11}$ M. Richter, P. Marquetand, J. Gonzalez-Vázquez, I. Sola, and L. Gonzalez, J. Phys. Chem. Lett. 3, 3090 (2012).

${ }^{12}$ L. Martinez-Fernandez, I. Corral, G. Granucci, and M. Persicob, Chem. Sci. 5, 1336 (2014).

${ }^{13}$ R. S. Minns, D. S. N. Parker, T. J. Penfold, G. A. Worth, and H. H. Fielding, Phys. Chem. Chem. Phys. 12, 15607 (2010).

${ }^{14}$ X. Qiu, C. Qin, J. Wang, Y. Tang, and B. Zhang, Phys. Rev. A 86, 032505 (2012).

${ }^{15}$ Y. Liu, G. Knopp, Y. Sych, P. Radi, and T. Gerber, Phys. Chem. Chem. Phys. 15, 18101 (2013).

${ }^{16}$ Y. Liu, T. Gerber, Y. Sych, P. Radi, and G. Knopp, Opt. Express 21, 16639 (2013).

${ }^{17}$ A. T. J. B. Eppink and D. H. Parker, Rev. Sci. Instrum. 68, 3477 (1997).
${ }^{18}$ Y. Liu, T. Gerber, P. Radi, Y. Sych, and G. Knopp, Chem. Phys. Lett. 610-611, 153 (2014).

${ }^{19}$ A. M. Walser, M. Meisinger, P. Radi, T. Gerber, and G. Knopp, Phys. Chem. Chem. Phys. 11, 8456 (2009).

${ }^{20} \mathrm{H}$. Studzinski, Ph.D. thesis, University of Kiel, 2007.

${ }^{21}$ T. Gerber, Y. Liu, G. Knopp, P. Hemberger, A. Bodi, P. Radi, and Y. Sych, Rev. Sci. Instrum. 84, 033101 (2013).

${ }^{22}$ A. Held, H. L. Selzle, and E. W. Schlag, J. Phys. Chem. A 102, 9625 (1998).

${ }^{23}$ V. Blanchet, M. Z. Zgierski, T. Seideman, and A. Stolow, Nature 401, 52 (1999).

${ }^{24}$ A. Stolow, A. E. Bragg, and D. M. Neumark, Chem. Rev. 104, 1719 (2004).

${ }^{25}$ E. L. Hommel and H. C. Allen, Analyst 128, 750 (2003).

${ }^{26} \mathrm{~V}$. Myrseth, Cand. Scient. Thesis, University of Bergen, 2000.

${ }^{27}$ J. P. Maier and D. W. Turner, J. Chem. Soc., Faraday Trans. 2 69, 196 (1973).

${ }^{28}$ T. G. Blease, R. J. Donovan, P. R. R. Langridge-Smith, and T. Ridley, Laser Chem. 9, 241 (1988).

${ }^{29}$ M. Clara, Th. Hellerer, and H. J. Neusser, Appl. Phys. B 71, 431 (2000).

${ }^{30}$ E. Yamamoto, T. Yoshidome, T. Ogaw, and H. Kawazumi, J. Electron Spectrosc. Relat. Phenom. 63, 341 (1993).

${ }^{31}$ J. H. Callomon, J. E. Parkin, and R. Lopez-Delgado, Chem. Phys. Lett. 13, 125 (1972).

${ }^{32}$ A. E. W. Knight, C. S. Parmenter, and M. W. Schuyler, J. Am. Chem. Soc. 97, 1993 (1975)

${ }^{33}$ A. E. W. Knight, C. S. Parmenter, and M. W. Schuyler, J. Am. Chem. Soc. 97, 2005 (1975).

${ }^{34}$ J. L. Knee, C. E. Otis, and P. M. Johnson, J. Phys. Chem. 87, 2232 (1983).

${ }^{35}$ J. Hofstein, H. Xu, T. Sears, and P. M. Johnson, J. Phys. Chem. A 112, 1195 (2008).

${ }^{36}$ Y. Liu, B. Tang, H. Shen, S. Zhang, and B. Zhang, Opt. Express 18, 5791 (2010). 